\title{
Investigation of the influence of the positive reinforcement method using biofeedback
}

\begin{abstract}
This article is about research in the field of biofeedback. Two methods of generating positive reinforcement are considered. The results of the influence of each of them are presented. Also the results of initial testing on a group of healthy speakers are presented
\end{abstract}

Keywords: speech, rehabilitation, training, biofeedback, positive reinforcement

\author{
Volume 2 Issue 2 - 2017
}

\author{
Kharchenko SS \\ Tomsk State University of Control Systems and \\ Radioelectronics, Russia
}

\begin{abstract}
Correspondence: Kharchenko SS, Tomsk State University of Control Systems and Radioelectronics, Tomsk city, Russia, Emailss.kharchenko@gmail.com
\end{abstract}

Received: March 15, 2017 | Published: March 24, 2017

\section{Introduction}

One of the most severe complications after surgical treatment of larynx malignant neoplasms is a full loss of sonorous speech and a violation of the respiratory system. The especially critical problem arises with the voice communication of working-age patients of between 40 and 60 years of age, impeding and restricting their social, labor and professional opportunities. The aiming of scientists to solve the problem of improving the life of cancer patients in a comprehensive manner, which largely depends on how successfully the speech recovery process was, is absolutely justified. According to opinion of many authors the key method of the speech restoration after laryngeal complete dissection is logopedic-esophageal speech forming. ${ }^{1-4}$ Tomsk Cancer Research Institute has been employing software tools in the process of the voice rehabilitation of oncologic patients after surgical service of laryngeal and laryngopharyngeal cancer for already fifteen years. The methods based on the biofeedback are used on each stage. Principle of the method of biofeedback consists in visualization of current physiological values, determined by a clinical protocol on a monitor screen or any other output devices for a patient. A protocol expresses as a set of conditions, specifying biofeedback procedure carrying-out. All biofeedback protocols are divided into two large groups:

1) "Neurofeedback"-which presents a movement, qualified in English-language literatures, in terms of which modification of different parameters of electroencephalogram (EEG) of the brain (amplitude, intensity, EEG basic rhythm coherence - which expresses via definition of "neurotherapy");

2) "Biofeedback"-which expresses a way in terms of which indicators of the vegetative activation, such as skin conductivity, cardiogram, heart rate, breathing, electromyogram, temperature, photoplethysmogram, base frequency and others undergo changes.

In the current work we will examine biofeedback (BFB) in the frame of it's the second exceptional method type. Means of reinforcement also act an important part in BFB-based processes and methods. There are three types of reinforcement:

A. Positive reinforcement-video record watching, playing of luscious music and other positive forms, drawing forth positive emotions, are used as a reinforcement;

B. Negative reinforcement-forms, drawing forth any negative reactions, such as image deformations, unpleasant music when wrong performance of the task, are used for this kind of the reinforcement.

C. Mixed reinforcement-using of both positive and negative reinforcements depending on the train task completion/failure.

Each of reinforcement methods has the right to be used and has its own positive features basing on opinions of different authors. Nevertheless presently there is no decisive answer which method is the most effective. Methods converting information obtained into static visual patterns are often used presently in Russia in software and hardware-software systems with biofeedback in the process of rehabilitation. Basing on the results of the methods used rehabilitation averaged term is about 4-5 weeks and the results are not always positive. In some way it is associated with the use of static visual patterns only. During visual signaling the sound cuts response time by $5-10 \%$ as it is shown in researches on audio-visual perception. ${ }^{5}$ This result presents sound ability to influence visual perception. Consequently the use of the audio-visual push acting as a positive reinforcement instead of static visual patterns allows improving of training quality that is speeding up the process of habituation to rehabilitation actions. In future it could correct existing method in such a way that a rehabilitation term would be reduced or training results would be better within the same term limits. As there are some patients for whom 4-5 weeks are not enough for a complete rehabilitation, method changes provided may take place. It is also should be mentioned that the use of the audio-visual pushes can help to reduce patients' psychoemotional tension in the rehabilitation process if it is a correct choice of audio component. Patient's psychoemotional state is one of the most important elements of rehabilitation as it is a psychosis in its different manifestations which is contraindication to BFB-based methods. In the way of audio components of audio-visual pushes it is recommended to use sound close to classical music. First of all it is connected with the results of the research that such kind of music makes positive impact on people of 30 years and older. ${ }^{6}$ Overall condition of test persons increased, particularly level of activity and emotional state were up with anxious feeling being down. As noted by logopedists working with patients on the rehabilitation period, feeling of foreboding often appears after partial or complete surgical removal of larynx (Laryngectomy) which gets in the way of trainings. Reduction of anxious feeling of the patients could positively influence the process of rehabilitation and trainings based on biofeedback. 
For checking of hypothesis for a positive impact of audio-visual positive reinforcement in BFB-based methods instead of static patterns the following experiment was performed. Software system making it possible to determine announcer's base frequency in real-time mode was used for the current experiment. 10 healthy announcers, men and women of 23-30 years old were involved into the experiment. In the first part of the experiment it was required from announcers to increase upper range limit of the voice when software system working mode with the use of static patterns during a week as a reinforcement. After a month the same announcers were involved into a week training process where audio-visual elements were used as reinforcement (Tables 1) (Table 2). Growth of upper range limit of the announcer's voice as a result of the experiment is rated in percentage terms. The following results were obtained:

a) 6 of 10 announcers showed the best results when using audiovisual reinforcement.

b) 3 of 10 announcers showed the best results when using static reinforcement but one of three participants had a respiratory disease before the second week of the experiment which could influence the results for the announcer.

c) 1 of 10 announcers showed the same results during the first and the second weeks of the experiment.

Table I Results of the first experimental week on influence of the reinforcement in BFB-based methods

\begin{tabular}{lllllllllll}
\hline Patient & \multicolumn{7}{c}{ The results of training, Hz (Median) } \\
\hline Identifier & Sex & I & $\mathbf{2}$ & $\mathbf{3}$ & $\mathbf{4}$ & $\mathbf{5}$ & $\mathbf{6}$ & $\mathbf{7}$ & $\begin{array}{l}\text { Progress, I-7 days, } \\
\text { increase in \% }\end{array}$ \\
\hline Abr. & female & 163 & 165 & 167 & 166 & 168 & 168 & 169 & 3,68 \\
Dub. & male & 120 & 124 & 125 & 125 & 124 & 126 & 126 & 5,00 \\
Kor. & male & 113 & 117 & 116 & 118 & 118 & 120 & 121 & 7,08 \\
Kha. & male & 134 & 139 & 139 & 142 & 141 & 144 & 145 & 8,21 \\
Kum. & female & 167 & 167 & 168 & 169 & 170 & 170 & 171 & 2,40 \\
Vol. & female & 171 & 172 & 172 & 173 & 174 & 174 & 174 & 1,75 \\
Opl. & female & 165 & 168 & 170 & 172 & 171 & 172 & 171 & 3,64 \\
Ben. & male & 121 & 121 & 122 & 122 & 124 & 125 & 125 & 3,31 \\
Smi. & male & 123 & 123 & 124 & 126 & 124 & 127 & 128 & 4,07 \\
Korl. & male & 140 & 141 & 142 & 144 & 145 & 147 & 146 & 4,29 \\
\hline
\end{tabular}

Table 2 Results of the second experimental week on influence of the reinforcement in BFB-based methods

\begin{tabular}{llllllllll}
\hline Patient & \multicolumn{7}{c}{ The results of training, Hz (Median) } \\
\hline Identifier & Sex & I & $\mathbf{2}$ & $\mathbf{3}$ & $\mathbf{4}$ & $\mathbf{5}$ & $\mathbf{6}$ & $\mathbf{7}$ & $\begin{array}{l}\text { Progress, I-7 days, } \\
\text { increase in \% }\end{array}$ \\
\hline Abr. & female & 164 & 166 & 167 & 167 & 169 & 171 & 171 & 4,27 \\
Dub. & male & 120 & 123 & 124 & 124 & 126 & 125 & 127 & 5,83 \\
Kor. & male & 114 & 114 & 116 & 115 & 119 & 121 & 120 & 5,26 \\
Kha. & male & 133 & 135 & 136 & 136 & 139 & 142 & 146 & 9,77 \\
Kum. & female & 168 & 167 & 169 & 170 & 173 & 172 & 174 & 3,57 \\
Vol. & female & 172 & 172 & 174 & 173 & 175 & 175 & 177 & 2,91 \\
Opl. & female & 167 & 169 & 170 & 173 & 174 & 171 & 174 & 4,19 \\
Ben. & male & 124 & 124 & 125 & 125 & 127 & 126 & 127 & 2,42 \\
Smi. & male & 124 & 124 & 125 & 126 & 126 & 127 & 129 & 4,03 \\
Korl. & male & 140 & 140 & 143 & 143 & 142 & 143 & 145 & 3,57 \\
\hline
\end{tabular}

\section{Conclusion}

In general this experiment revealed relevance of the use of audiovisual positive reinforcement instead of a static one. Experiment results can be applied for patients with larynx surgical removal whereas the experiment was carried out on healthy announcers as vegetative processes of healthy people and patients do not change.

\section{Acknowledgements}

The study was performed by a grant from the Russian Science
Foundation (project № 16-15-00038)

\section{Conflict of interest}

The author declares no conflict of interest.

\section{References}

1. Balatskaya LN. Speech rehabilitation and quality of life after treatment of patients with head and neck tumors [in Russian. Thesis for the degree of doctor of biological sciences, Tomsk Scientific Center, Tomsk, Russia: physiology Oncology research institute of oncology; 2001. 269 p. 
2. Dietz JH. Adaptive rehabilitation in cancer. A program to improve quality of survival. Postrad Med. 1980;68(1):145-147.

3. Hirano M. Recent advances in rehabilitation of head and neck cancer patients. Otorhinolaringol. 1990;17(4 Pt 2):795-801.

4. Brodnitz FS. Psychologycal considerations in vocal rehabilitation. $J$ Speech Hear Disord. 1981;46:21-26.
5. Wang Y, Celebrini S, Trotter Y, et al. Visuo-auditory interactions in the primary visual cortex of the behaving monkey: Electrophysiological evidence. BMC Neuroscience. 2008;9:79.

6. Yerkinbekova Meyrim Anarbekovna, Kasumova Roza Sadukovna, Shagurbaeva Mentay Dogdurbaevna, et al. Study of the influence of different styles of music on young people's mental States. Austrian Journal of Humanities and Social Sciences. 2014;1:276-290. 